\title{
Study on the bulk grain auto-loading system based on the machine vision technology
}

\author{
Xu Zengpu ${ }^{\mathrm{a}}$, Zhang $\mathrm{chi}^{\mathrm{b}}$, Wang Yongqiang ${ }^{\mathrm{c}}$, Zhou Congling ${ }^{\mathrm{d}}$ \\ Department of Mechanical Engineering,Tianjin University of Science and Technology:No.1038 Dagu \\ Nanlu,Hexi District, Tianjin,China,300222 \\ axuzp@tust.edu.cn, ${ }^{\mathrm{b}}$ zhangchi1025@163.com, ${ }^{\mathrm{c}}$ wangyq@tust.cn, ${ }^{\mathrm{d}}$ zhoucling@tust.edu.cn
}

Keywords: Bulk grain loading, Machine vision, Image processing, Canny algorithm

\begin{abstract}
This paper designs a kind of bulk grain auto-loading system based on the machine vision technology, introduces the hardware composition of system, sketches the collection process of the real-time images, and uses the Canny algorithm to process and analyze the images. It is thus clear that this system is able to improve the efficiency and accuracy of the bulk grain loading operation as well as reduce the labor intensity of workers.
\end{abstract}

\section{Introduction}

It is the process for the traditional bulk grain loading operation that worker observe the height and volume of the material stack in the carriage by eyes, judge whether the truck bed is filled or not depending on the experience, manually control switch valve, and prompt vehicle to move forward through blowing the whistle(Figure 1). This kind of control way does not only influence the efficiency of loading, but may also cause the incorrect operation by the operator fatigue, and then appears the phenomena such as over-shipment or over-loading. At the same time, the work environment with the serious dust pollution is bad for the staff health to some degree ${ }^{[1]}$.

During this operation process, the needed information such as vehicle location, moving distance, the material stack height in the carriage, etc., mostly comes from the visual observation of the operators. These real-time information observed by human eyes can be detected and obtained by using the modern technological means such as machine vision, thus automatically opening and closing feeding valve can be realized with the help of software, the loud speaker is used to prompt the driver to move vehicles, and then the process is recycled until the entire process is completed. Practices show that compared with the traditional operation ways, loading efficiency can be increased by $20 \%$ by using the system for bulk grain loading operation, the over-loading phenomenon is completely eradicated, and the operation efficiency and accuracy of the bulk grain loading is effectively improved.

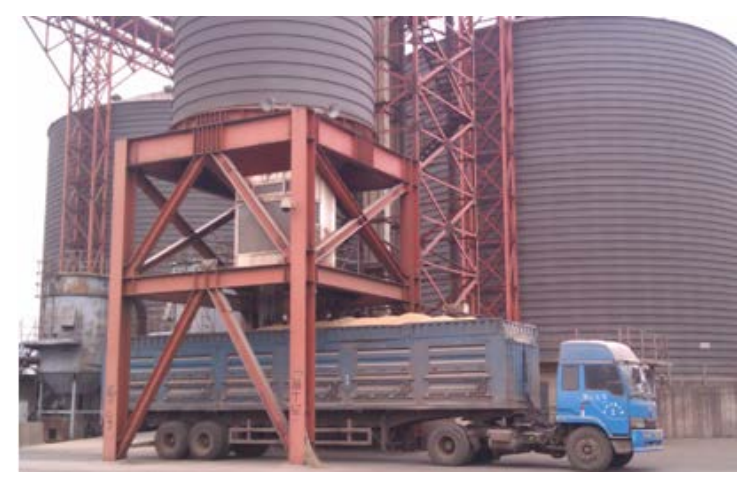

Figure 1. Bulk grain loading operation spot

\section{System composition and working theory}

This system is composed of a CCD camera, light source, loudspeaker, image acquisition card, industrial computer, and so on, as shown in Figure 2. It is the working principle that after the vehicle 
waiting for loading parks in the initial discharge location (planned initial parking location), the driver presses the start button, the camera and light source equipment installed on the buffer hopper steel structure immediately start, feeding valve opens with time delay, and the feeding outlet automatically conducts discharge for loading.

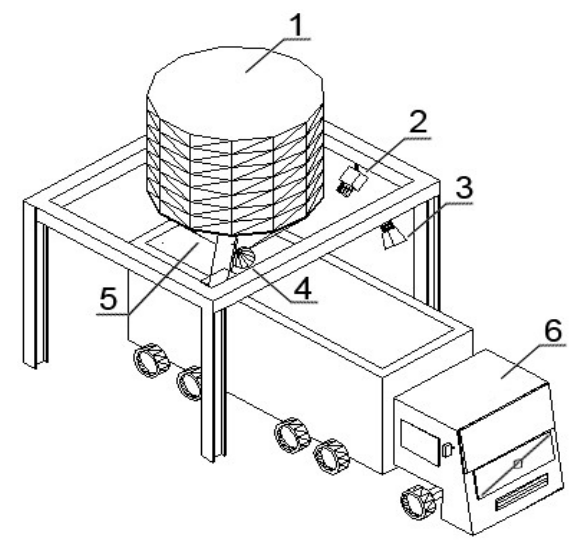

Figure 2. System hardware composition

1-Buffer bucket； 2-CCD camera； 3- Loudspeaker; 4- Light source;

5- Discharge outlet ; 6-Vehicle to be loaded

Camera is used to collect the real-time images of change of material height in the boxcar, after the conversion of light signal and analog signal, the real-time images are sent to industrial computer though the image acquisition card. The computer technically processes the obtained real-time images with the help of professional softwares to identify the amount of material information in the loading process. This is adopted as the decision-making basis for controlling opening and closing of valve, and the loudspeaker is used to prompt driver to move vehicle forward, until finishing the whole loading process.

\section{Image processing}

\subsection{Image preprocessing}

The vehicles are relatively static as loading because the industrial camera position is fixed. Hence, the carriage wall can be regarded as the background, and the artesian material is regarded as the moving target. When an arbitrary pile of material flows, the background in the image doesn't obviously change[2], so the background subtraction can be used for the pre-segmentation of target and background, thus the images can be simplified initially. Due to the better gravity flow of bulk grain materials, the upper surface of pile material can be seen as a stable flow line L1, and the top edge of the carriage can be regarded as a relatively fixed straight line L2. As the material level is continuously rising, the distance $\mathrm{d}$ between L1 and L2 gradually decreases. When $\mathrm{d}$ is close to the safe distance D0, the system automatically close the valve, and stops loading. Thus it can be seen that the key for image processing is to find the material flow line L1 and the top edge of the carriage line $\mathrm{L} 2$, and then calculate the distance between them.

\subsection{Edge extraction based on Canny algorithm}

The traditional edge extraction methods include Roberts operator, Sobel operator, Prewitt operator, and so on. These operators have the advantages such as good real-time performance, simple operation. But they are more sensitive to the noise and are difficult to distinguish noise and image edge, which is likely to cause the missing detection or false detection, and the detected images are not fine enough with the edge blur. Due to the scene environment complexity of loading, it is necessary to take into account the effect of the factors such as background noise factors (such as carriage vibration, dust, etc.), the change of light ray (different light conditions in the different periods), the weather changes 
(different weather conditions of rain, snow, fog, etc), the shadow of carriages and the surrounding objects, sundries, and so on, on the collection image quality. Therefore, it is difficult to get clear edge images by using traditional detection operator.

The Canny operator based on optimization algorithm is used in this paper, and is widely applied due to its good edge detection precision and stronger antinoise performance. The realization of the Canny operator mainly include four steps such as smooth filtering, compute the gradient magnitude and direction, gradient Non-Maximum Suppression, detection and edge connection of double-threshold method.

\section{Step 1: smooth filtering}

To construct filter $\mathrm{G}(\mathrm{x})$, The convolution operation is conducted on the original image according to the row and column, respectively, to get the smooth image $I(x, y)$.

$$
G(x)=\frac{\exp \left(-x^{2} / 2 \sigma^{2}\right)}{2 \pi \sigma^{2}}
$$

where $\sigma$ is the standard deviation of gaussian function, and is used to control the smooth degree.

\section{Step 2: compute the gradient magnitude and direction}

The finite difference of first-order partial derivatives with the $2 \times 2$ neighborhood is used to calculate the gradient magnitude $^{M(\mathrm{x}, \mathrm{y})}$ and the gradient direction $H(\mathrm{x}, \mathrm{y})$ of the image $I(\mathrm{x}, \mathrm{y})$ after the smoothness, and the equations are expressed as:

$$
\left\{\begin{array}{l}
M(\mathrm{x}, \mathrm{y})=\sqrt{k_{x}^{2}(\mathrm{x}, \mathrm{y})+\mathrm{k}_{y}^{2}(\mathrm{x}, \mathrm{y})} \\
H(\mathrm{x}, \mathrm{y})=\arctan \left[\mathrm{k}_{x}(\mathrm{x}, \mathrm{y}), \mathrm{k}_{y}(\mathrm{x}, \mathrm{y})\right]
\end{array}\right.
$$

Where $k_{x}, k_{y}$ are the results of image $I(\mathrm{x}, \mathrm{y})$ which is acted by the filters of $f_{x}, f_{y}$ according to the row and column.

\section{Step 3: Non-Maximum Suppression is conducted on the gradient magnitude.}

The purpose of Non-Maximum Suppression for the gradient magnitude is to find all of the possible edge points of $I(\mathrm{x}, \mathrm{y})$. The Canny algorithm uses a $\quad 3 \times 3$ neighbourhood to act on the total pixels of the gradient magnitude array $M(\mathrm{x}, \mathrm{y})$, and then conducts the gradient amplitude of interpolation along the gradient direction. The neighborhood center element $M(\mathrm{x}, \mathrm{y})$ is compared with the interpolation results of the two gradient amplitudes to find the edge points.

\section{Step 4: detection and edge connection of double-threshold method}

The two thresholds edge image $H(i, j)$ and $L(i, j)$ are obtained by using the two thresholds of high threshold and low threshold ( $H_{\text {th }}$ and $L_{\text {th }}$ ) to segment. The edge is connected into a contour in the high threshold image $H(i, j)$, when connecting to the endpoint, the weak edge points is searched in the low threshold image $L(i, j)$ to the edge interspace in the high threshold image $H(i, j)$. Until all the edge points in the image are connected, finally, the edge image is obtained. 


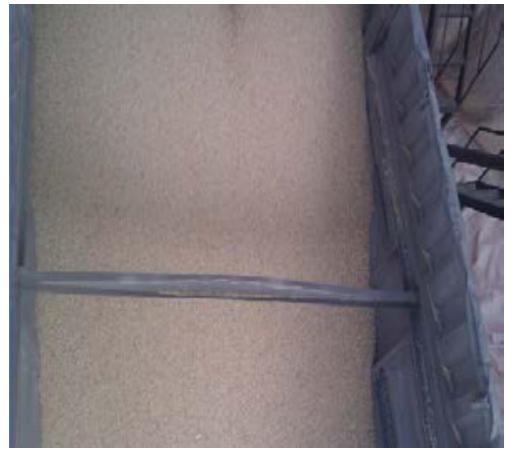

(a) Original image

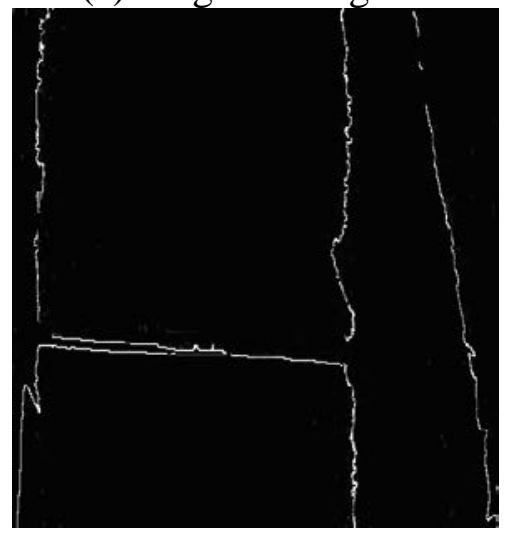

(c) Canny processing

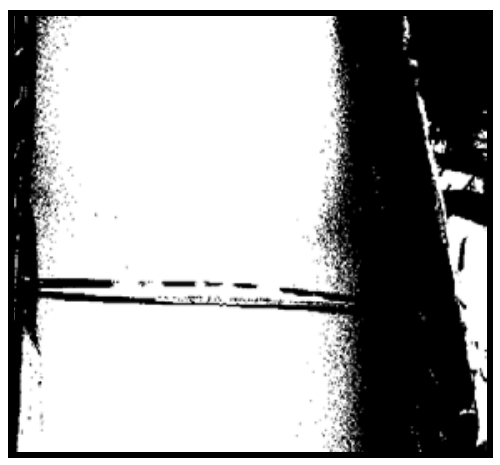

(b) Two-value processing result

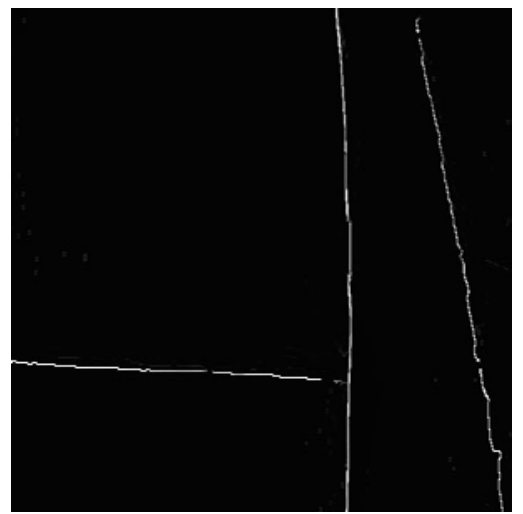

(d) Final processing results

Figure 3. Image processing results

\section{Experimental results and comparison}

It can be seen from Figure 3 that both the clarity and continuity of the results obtained by the Canny algorithm can satisfy the precision requirement. We randomly selected 10 images in the process of loading, carry out the edge detection for images by the Canny algorithm, and compare the read highest point of material and the distance data of the top edge of the carriage with the actually measured data at this moment.

Table1. The comparision between the experimental data and measured data

\begin{tabular}{|c|c|c|c|c|}
\hline No. & $\begin{array}{c}\text { Canny Processing data } \\
(\mathrm{cm})\end{array}$ & $\begin{array}{c}\text { Actually measured data } \\
(\mathrm{cm})\end{array}$ & $\begin{array}{c}\text { Absolute error } \\
(\mathrm{cm})\end{array}$ & $\begin{array}{c}\text { Error percentage } \\
(\%)\end{array}$ \\
\hline 1 & 81.3 & 81.1 & 0.2 & 0.246 \\
\hline 2 & 65.5 & 65.3 & 0.3 & 0.306 \\
\hline 3 & 53.9 & 53.6 & 0.1 & 0.559 \\
\hline 4 & 50.2 & 50.3 & 0.0 & 0.199 \\
\hline 5 & 48.4 & 48.4 & 0.1 & 0.000 \\
\hline 6 & 44.1 & 44.0 & 0.1 & 0.227 \\
\hline 7 & 42.6 & 42.5 & 0.1 & 0.252 \\
\hline 8 & 39.8 & 39.7 & 0.0 & 0.000 \\
\hline 9 & 39.2 & 39.2 & 0.0 & 0.000 \\
\hline 10 & 30.9 & 30.9 & & 0.2 \\
\hline
\end{tabular}


From the measured results, the maximum of measured absolute error is $0.3 \mathrm{~cm}$, and the safe distance between the highest point of material and the top edge of the carriage is about $15 \mathrm{~cm}$, so the effect of measurement error on loading precision and accuracy is very little. Via the practical inspection, this method is used to detect the height of bulk grain material level in the carriage, the automatic loading system which is realized by the automatic control operates stably, and its precision is reliable.

\section{Conclusions}

This paper puts forward a kind of bulk grain discharging loading system based on machine vision technology, introduces the overall design of the system, the hardware composition and the image processing method. The Canny algorithm adopted in study can better satisfy the working conditions and the precision requirement of bulk grain loading operation, improves the real-time performance and reliability of the system detection, and provides theory basis for the loading and unloading of automation of dry bulk such as food, ore, and so on. However, from the actual operation, there still exists interference factors such as vibration, dust, shadows, and etc., and system robustness, reliability and accuracy still have the room for improvement. Therefore, the follow-up work will mainly focus on improving the algorithm, enhancing algorithm adaptability, perfecting the system function, and further improving the safety and reliability of the loading.

\section{References}

[1] Yang lixin, Bulk grain mobile conveyor equipment and loading and unloading process in Beiliang Port. Harbour loading and unloading, 2006 (166): 11-12.

[2] Tian mingrui,Study on asphalt concrete discharging loading based on machine vision [D] Xi 'an: Chang 'an university, 2010.

[3] Ma songde, Zhang zhengyou, Computer vision [M]. Beijing: science press, 1998.

[4] Chen meilan, Machine vision key technology and application example analysis [J]. Journal of modern computer, 2006 (227): 21-25.

[5] Li gun, Yan fabao, Su yanrui,et al. Adaptive double-threshold oil level in tank of infrared imaging detection based on CANNY. [J]. Journal of electronic measurement and instrument, 2009 (23), 44-49.

[6] Wang guibin, Edge detection algorithm based on the fusion of canny operator and morphology

[D], Harbin: Master’s degree thesis, 2014. 